\title{
On the Chow group of zero-cycles of Calabi-Yau hypersurfaces
}

\author{
Ivan Bazhov \\ Institut de Mathématiques de Jussieu
}

\begin{abstract}
We prove the existence of a canonical zero-cycle $c_{X}$ on a Calabi-Yau hypersurface $X$ in a complex projective homogeneous variety. More precisely, we show that the intersection of any $n$ divisors on $X, n=\operatorname{dim} X$ is proportional to the class of a point on a rational curve in $X$.
\end{abstract}

\section{Introduction}

The Chow groups $\mathrm{CH}_{i}(X)$ are abelian groups generated by classes of algebraic cycles modulo rational equivalence, they are basic invariants of an algebraic variety. One has $\mathrm{CH}_{n}(X)=\mathbb{Z}[X]$ and $\mathrm{CH}_{n-1}=\operatorname{Pic}(X)$ for a smooth variety $X$ of dimension $n$, hence these two groups are well understood. Concerning the group of zero-cycles, it is known (cf. $[8,9])$ that $\mathrm{CH}_{0}(X)$ is infinite dimensional if $h^{\operatorname{dim} X}\left(X, \mathcal{O}_{X}\right)>0$, and the other Chow groups are even more mysterious. Our motivating point is the following theorem, which contrasts with the results of $[8,9]$.

Theorem 1.1 ([2]). Let $X$ be a K3 surface.

a. All points of $X$ which lie on some (possibly singular) rational curve have the same class $c_{X}$ in $\mathrm{CH}_{0}(X)$.

b. The image of the intersection product

$$
\operatorname{Pic}(X) \otimes \operatorname{Pic}(X) \rightarrow \mathrm{CH}_{0}(X)
$$

is contained in $\mathbb{Z} c_{X}$.

c. The second Chern class $c_{2}(X) \in \mathrm{CH}_{0}(X)$ is equal to $24 c_{X}$. 
From now on $\mathrm{CH}^{i}(X)=\mathrm{CH}_{n-i}(X) \otimes \mathbb{Q}$. In the paper [1] Beauville put the theorem above in a more general framework and proposed a conjectural explanation for hyper-Kähler manifolds: the class map cl : $\mathrm{CH}^{i}(X) \rightarrow H^{2 i}(X)$ is injective on the subring generated by divisors. A stronger version, which involves Chern classes of tangent bundles, was formulated in [11]. The reader can find particular results about hyper-Kähler manifolds in [5, 7, 12, 13].

An example in [1] shows that the class map may be not injective on the ring generated by divisors if $X$ is a Calabi-Yau variety. Fortunately, this example deals with cycles of positive dimension and still there is a hope that the class map is injective on zero-cycles or, equivalently, that the intersection of any $\operatorname{dim}(X)$ divisors on $X$ is proportional to a canonical zero-cycle $c_{X}$ in $\mathrm{CH}_{0}(X)$. The existence of such a cycle is trivial if $\operatorname{Pic}(X)$ is generated by one element $H$ : we can put $c_{X}=H^{\operatorname{dim} X} / \operatorname{deg}\left(H^{\operatorname{dim} X}\right)$. We aim to show the existence of $c_{X}$ prove the following analog of the Theorem 1.1 at least for some Calabi-Yau varieties with higher Picard rank.

Theorem 1.2. Let $Y$ be a complex projective homogeneous variety of dimension $n+1$ and $X$ be a general element of the anti-canonical system $\left|-K_{Y}\right|$ on $Y$.

a. There exist a constant cycle subvariety (cf. Definition 3.1) of positive dimension. All points of $X$ which lie on such constant cycle variety have the same class $c_{X}$ in $\mathrm{CH}_{0}(X)$.

b. The image of the intersection product

$$
\operatorname{Pic}(X)^{\otimes n} \rightarrow \mathrm{CH}_{0}(X)
$$

is contained in $\mathbb{Z} c_{X}$.

c. The top Chern class $c_{n}(X) \in \mathrm{CH}_{0}(X)$ is proportional to $c_{X}$.

The proof uses a notion of constant cycle subvarieties, which was introduced in [6] and used in [7, 13] to study $\mathrm{CH}_{0}(X)$ for hyperKähler manifolds. The main point of our proof is an existence of a constant cycle divisor in an ample class (in the situation of K3 surface, the analog is the existence of rational curves in an ample class). Our result (b) also shows that the restriction to our Calabi-Yau hypersurface of any curve in a homogeneous space is proportional to the canonical cycle $c_{X}$ (cf. Proposition 4.1). Indeed, by the hard Lefschetz theorem, any curve class in $Y$ is an intersection of divisor 
classes in $Y$ and furthermore the cycle class $\mathrm{CH}^{n}(Y) \rightarrow H^{2 n}(Y)$ is injective, so any element of $\mathrm{CH}^{n}(Y)$ is an intersection of divisors.

The text is organized as follows. Firstly, we briefly recall basic facts about homogeneous spaces. Then, we construct a constant cycle divisor associated with an effective class of curves in $Y$. Using these divisors we finish the proof in the last section.

Acknowledgement. I am grateful to my advisor Claire Voisin for her kind help and patient guidance during the work.

\section{Homogeneous varieties and their properties}

We recall some basic facts about homogeneous spaces (see [3] and the references therein). For a projective homogeneous variety $Y$ the cone of effective divisors $\operatorname{Eff}(Y)$, which is the closure of the cone of ample divisors, is a polyhedral cone. Every rational point in it represents a globally generated line bundle, which is ample if and only if the point belongs to the interior of $\operatorname{Eff}(Y)$. Since $Y$ is Fano, its anti-canonical class $-K_{Y}$ belongs to the interior of $\operatorname{Eff}(Y)$.

For every face $\sigma \subset \operatorname{Eff}(Y)$ of codimension one, one defines the extremal contraction

$$
Y \stackrel{f_{\beta}}{\longrightarrow} Y_{\beta},
$$

of the primitive class $\beta \in \sigma^{\perp}$ of curves, where $\beta$ is the unique integral positive generator of the rational line $\sigma^{\perp}$, and $Y_{\beta}$ is a homogeneous variety of a smaller dimension and $f_{\beta}$ is a fibration. There is the natural identification

$$
\beta^{\perp}=f_{\beta}^{*}\left(\operatorname{Pic}\left(Y_{\beta}\right)\right) \subset \operatorname{Pic}(Y) .
$$

Vice versa, for any extremal ray $\mathbb{R}_{>0} \beta$ there is a face $\sigma=\beta^{\perp} \cap \operatorname{Eff}(Y)$ of codimension one and we can define a contraction $f_{\beta}$. We denote the set of all primitive effective classes of curves on extremal rays by $\mathcal{R}$.

A general fiber $Y_{0}$ of $f_{\beta}$ is a generalized Grassmann variety in the following sense: it is a homogeneous space $G / P$, where $P$ is a maximal parabolic subgroup of $G$. We use only one fact: $\operatorname{Pic}\left(Y_{0}\right)$ is generated by the class of an ample bundle $\mathcal{O}_{Y}(1)$ and the unique primitive effective class $\beta \in \mathrm{CH}_{1}\left(Y_{0}\right)$ has degree 1 with respect to it (we call $\beta$ the class of a line). 


\section{Constant cycle divisors}

The notion of constant cycle subvarieties was introduced in [6] and used in [7] and [13]. The goal of this section is to construct constant cycle divisors on Calabi-Yau hypersurfaces.

Definition 3.1. A subvariety $Z \subset X$ of positive dimension is called a constant cycle subvariety if for any two points $z, z^{\prime} \in Z$ one has $[z]=\left[z^{\prime}\right]$ in $\mathrm{CH}_{0}(X)$. If, moreover, $\operatorname{codim}_{X}(Z)=1$, we call $Z$ a constant cycle divisor (or CCD for short). We will denote by $c_{Z} \in \mathrm{CH}_{0}(X)$ the common class of the points $z \in Z$.

Example 3.2. Let $\pi: X \rightarrow \mathbb{P}^{n}$ be a ramified double cover. Clearly, the class in $\mathrm{CH}_{0}(X)$ of the degree two cycle $x_{1}+x_{2}=\pi^{-1}(p)$ does not depend on the choice of $p \in \mathbb{P}^{n}$, and if, moreover, $x_{1}$ and $x_{2}$ coincide, then the class $\left[x_{1}\right]=\left[x_{2}\right]$ is determined in $\mathrm{CH}_{0}(X)$ uniquely. The locus, where the two preimages $x_{1}$ and $x_{2}$ coincide is the divisor $D$ of ramification. So, $D$ is CCD on $X$.

The rest of the section is to expand the idea of the last example: having a family of zero-cycles on $X$, all of the same class and degree, we are looking for cycles represented by one point (with the corresponding multiplicity).

Construction 3.3. Let $Y$ be a Fano variety of dimension $n+1$ and assume that $Y$ has trivial $\mathrm{CH}_{1}(Y)$ group, which means the following.

(*) The natural class map

$$
\mathrm{cl}: \mathrm{CH}_{1}(Y) \otimes \mathbb{Q} \longrightarrow H^{2 n}(Y, \mathbb{Q})
$$

is injective.

(Varieties with trivial Chow groups include projective homogeneous spaces, smooth toric varieties, and varieties admitting a stratification by affine spaces.)

Let $\beta$ be any effective class in $\mathrm{CH}_{1}(Y)$ and $M_{\beta}$ be the space of all (irreducible) rational curves $C \subset Y$ representing class $\beta$. Denoting by $\mathcal{C}$ the universal curve we have the natural diagram:

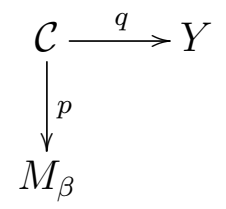


where $p$ and $q$ are the natural projections.

For a given hypersurface $X \subset Y$ we can construct the following variety:

$$
V_{X, \beta}=\{(C, x) \in \mathcal{C}: C \cap X=\operatorname{deg}(C \cap X) \cdot x\},
$$

where the quality is the equality of zero-cycles on $C$ (or even of subschemas of $C$ if $C$ is smooth). The subvariety $p\left(V_{X, \beta}\right)$ in $M_{\beta}$ describes all curves intersecting $X$ in one point with the maximal multiplicity. Due to our assumption $(\star)$, the class of the zero-cycle $\left.C\right|_{X}$ in $\mathrm{CH}_{0}(X)$ does not depend on the point $C$ in $M_{\beta}$. By [10], a family of torsion cycles in a variety has to be constant, hence the subvariety $q\left(V_{X, \beta}\right) \subset X$ is a constant cycle subvariety.

If $X \in\left|-K_{Y}\right|$ is general, so that $X$ is a smooth Calabi-Yau hypersurface in $Y$, and furthermore there is no obstruction for deformation of curves in $Y$, then denoting $d=\operatorname{deg} C \cap X$ we have

$$
\begin{array}{r}
\operatorname{dim} V_{X, \beta} \geq \operatorname{dim} M_{\beta}-(d-1)=\left(-K_{Y} \cdot \beta+(n+1)-3\right)-(d-1) \\
=(X \cdot C+\operatorname{dim} X-2)-d+1=\operatorname{dim} X-1,
\end{array}
$$

and we expect that $\overline{q\left(V_{X, \beta}\right)}$ is a CCD on $X$.

Notation 3.4. Continuing with settings of Construction 3.3, we put

$$
H_{\beta}=\overline{q\left(V_{X, \beta}\right)}
$$

Lemma 3.5. Let $Y$ be a projective homogeneous variety with Picard number one and dimension at least two and let $\beta$ be the class of a line. If $X \in\left|-K_{Y}\right|$ is general, then $H_{\beta}$ is a non-empty effective divisor on $X$.

Proof. First of all, $H_{\beta}$ can not be the whole of $X$, because it would imply that all points in $X$ are rationally equivalent. In order to show that $H_{\beta}$ is a non-empty effective divisor, it thus suffices to show that its virtual class is non-zero.

We denote the class of the standard polarization on $Y$ by $H_{1}$ and notation as in (1), let $\tilde{H}_{1}=q^{*} H_{1}$ be the corresponding divisor on the universal curve. We also put $H_{2}:=p_{*}\left(\tilde{H}_{1}^{2}\right)=p_{*} q^{*} H_{1}^{2}$, let $\tilde{H}_{2}=p^{*} H_{2}$. Denote by $K_{r e l}$ the class of the relative cotangent bundle on the universal curve, it is easy to see that $K_{r e l}=-2 \tilde{H}_{1}+\tilde{H}_{2}^{\prime}$, where $\tilde{H}_{2}^{\prime}=p^{*} H_{2}^{\prime}$ for some divisor $H_{2}^{\prime}$ on $M_{\beta}$. 
Let us show that $H_{2}=H_{2}^{\prime}$. By Grothendieck-Riemann-Roch formula,

$$
c_{1}\left(R^{0} p_{*} \mathcal{O}_{\mathcal{C}}\left(\tilde{H}_{1}\right)\right)=p_{*}\left(\tilde{H}_{1}^{2}+\frac{5}{6}\left(\tilde{H}_{1}^{2}-\tilde{H}_{1} \tilde{H}_{2}^{\prime}\right)+\frac{1}{12} \tilde{H}_{2}^{2}\right),
$$

but the left-hand side can be calculated geometrically: it is the locus of all curves $C$ in $M_{\beta}$ such that the restrictions of two sections in $H^{0}\left(Y, \mathcal{O}_{Y}\left(H_{1}\right)\right)$ to $C$ coincide. Hence the left-hand side is equal to $p_{*} q^{*}\left(H_{1}^{2}\right)=p_{*}\left(\tilde{H}_{1}^{2}\right)$. Since $p_{*}\left(\tilde{H}_{2}^{2}\right)=0$, we get

$$
p_{*}\left(\frac{5}{6}\left(\tilde{H}_{1}^{2}-\tilde{H}_{1} \tilde{H}_{2}^{\prime}\right)\right)=0 .
$$

Since $\tilde{H}_{1}$ has degree one on fibers of $p: \mathcal{C} \rightarrow M_{\beta}$, we get $p_{*}\left(\tilde{H}_{1} \tilde{H}_{2}^{\prime}\right)=$ $H_{2}^{\prime}$ and

$$
H_{2}^{\prime}=p_{*}\left(\tilde{H}_{1} \tilde{H}_{2}^{\prime}\right)=p_{*}\left(\tilde{H}_{1}^{2}\right)=H_{2} .
$$

The subvariety $V_{X, \beta} \subset \mathcal{C}$ is the intersection of $d$ divisors of classes $(0 \leq r \leq d-1)$

$$
d \tilde{H}_{1}+r K_{r e l},
$$

where the divisor number $r$ is to say that the $r$-th derivative of the restriction $\left.f_{X}\right|_{C}$ is zero at a point on the curve $C$, where $f_{X}$ is the defining equation for $X$; all together they mean that $\left.f_{X}\right|_{C}$ is one point with the maximal multiplicity. So, the degree of $H_{\beta}=q_{*}\left(V_{X, \beta}\right)$ can be calculated as the degree of the following intersection on $Y$ (we recall $\operatorname{dim} X=n, \operatorname{dim} Y=n+1$ ):

$$
\begin{array}{r}
H_{1}^{n-1} \cdot q_{*}\left[d \tilde{H}_{1} \cdot\left(d \tilde{H}_{1}+K_{r e l}\right) \cdot \ldots \cdot\left(d \tilde{H}_{1}+(d-1) K_{r e l}\right)\right] \\
=q_{*}\left[\tilde{H}_{1}^{n-1} \cdot d \tilde{H}_{1} \cdot\left(d \tilde{H}_{1}+K_{r e l}\right) \cdot \ldots \cdot\left(d \tilde{H}_{1}+(d-1) K_{r e l}\right)\right] \\
=q_{*}\left[d \tilde{H}_{1}^{n} \prod_{r=1}^{d-1}\left(d \tilde{H}_{1}+r\left(-2 \tilde{H}_{1}+\tilde{H}_{2}\right)\right)\right] \\
=q_{*}\left[d !\left(\tilde{H}_{1}^{n} \tilde{H}_{2}^{d-1}+\sum_{r=1}^{d-1}\left(\frac{d-2 r}{r}\right) \tilde{H}_{1}^{n+1} \tilde{H}_{2}^{d-2}\right)\right] .
\end{array}
$$

Clearly, points of $p_{*}\left(\tilde{H}_{1}^{n+1}\right)=p_{*} q^{*}\left(H_{1}^{n+1}\right)$ in $M_{\beta}$ correspond to curves which pass through a point of the intersection $H_{1}^{n+1}$ in $Y$. Since $H_{2}$ is ample on $M_{\beta}$, the intersection $\tilde{H}_{1}^{n+1} \tilde{H}_{2}^{d-2}$ is not empty. To finish the proof, we note that the sum $\sum_{r=1}^{d-1}(d / r-2)$ is strictly positive if $d>2$. 
Lemma 3.6. Let $Y$ be a homogeneous variety and $X \in\left|-K_{Y}\right|$ be general. If $f_{\beta}: Y \rightarrow Y_{\beta}$ is not a $\mathbb{P}^{1}$-fibration for some $\beta \in \mathcal{R}$, then $H_{\beta}$ is a non-empty effective $C C D$ on $X$.

Proof. It is enough to show that $V_{X, \beta}$ is non-empty of an expected dimension and a general fiber of $q: V_{X, \beta} \rightarrow q\left(V_{X, \beta}\right)$ is finite.

A general fiber $Y_{0}$ of $f_{\beta}: Y \rightarrow Y_{\beta}$ is a Schubert variety in $Y$, hence the restriction map $H^{0}\left(Y, \mathcal{O}_{Y}(X)\right) \rightarrow H^{0}\left(Y_{0},\left.\mathcal{O}_{Y}(X)\right|_{Y_{0}}\right)$ is surjective ([4, Section 3] or [3, Theorem 2.3.1]). Applying the previous lemma to $Y_{0}$ and $\left.X\right|_{Y_{0}}$ we see that $V_{X, \beta}$ is non-empty and projection $q$ has some finite fibers, hence a general fiber is finite. The dimension of $V_{X, \beta}$ is expected because the dimension of $M_{\beta}$ is expected.

Example 3.7. Let $Y=\mathbb{P}^{2} \times S$, where $S$ is a Fano variety of positive dimension and let $\beta$ be equal to $[l \times$ point $]$, where $l$ is a line in $\mathbb{P}^{2}$. If $X$ is a general Calabi-Yau hypersurface in $Y$ then the restriction of $H_{\beta}$ to a fiber of $\mathbb{P}^{2} \times S \rightarrow S$ is nine Weierstrass points on a plane elliptic curve. In particular, $H_{\beta}$ is non-empty and effective.

Example 3.8. Let $Y$ be as in Construction 3.3 and assume that

$$
f_{\beta}: Y \longrightarrow Y_{\beta}
$$

is a $\mathbb{P}^{1}$-fibration (again $\beta$ is the class of contracted curves, i.e, the class of a fiber). If $X$ is a general Calabi-Yau hypersurface then $H_{\beta}$ has class $\left.\left(-K_{Y}+K_{Y / Y_{\beta}}\right)\right|_{X}=\left.f_{\beta}^{*}\left(-K_{Y_{\beta}}\right)\right|_{X}$ in $\operatorname{Pic}(X)$ and $H_{\beta}$ is trivial on fibers of $f_{\beta}$ (in contrast to Lemma 3.6 and Example 3.7).

Example 3.9 (The curve of hyperflexes, cf. [6] and [14]). In this example $Y=\mathbb{P}^{3}, X$ is a generic quartic, and $\beta$ is the class of a line. The curve $C_{h f}=q\left(V_{X, \beta}\right)$ is a singular and irreducible curve in the linear system $\left|\mathcal{O}_{X}(20)\right|$ of geometric genus 201. In particular, $C_{h f}$ is not rational.

Geometrically, $C_{h f}$ arises as follows. Recall that for a quartic $X \subset \mathbb{P}^{3}$ a line $l \subset \mathbb{P}^{3}$ is called bitangent of $X$ if at every point $x \in X \cap l$ the intersection multiplicity is at least two, a bitangent $l$ is a hyperflex if there is a unique point of the intersection. We can consider the universal family of bitangents:

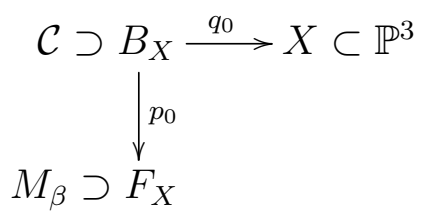


here $F_{X} \subset M_{\beta}$ is the subvariety of all bitangents and $B_{X}$ is the variety of pairs $(l, x)$, where $x$ is a point of contact of $l$ and $X$. The curve $C_{h f}$ can be defined as $q_{0}\left(p_{0}^{-1}\left(D_{h f}\right)\right)$, where $D_{h f}$ is the ramification divisor of the degree two map $p_{0}: B_{X} \rightarrow F_{X}$.

\section{The proof of Theorem 1.2}

Let us first prove the following.

Proposition 4.1. In the setting of Theorem 1.2, there exists a class $c_{X}$ in $\mathrm{CH}_{0}(X)$ such that the restriction $\left.C\right|_{X}$ of any curve in $Y$ is proportional to $c_{X}$ in $\mathrm{CH}_{0}(X)$.

Proof. Because of the linearity of the intersection, it is enough to prove the statement only for curves with class $\beta \in \mathcal{R}$. By construction of $H_{\beta}$, the corresponding zero-cycles can be represented by points (with multiplicities) on $H_{\beta}$ for $\beta \in \mathcal{R}$. Let us show that the class $c_{H_{\beta}}$ does not depend on the choose of $\beta \in \mathcal{R}$.

Case 1: $\operatorname{dim} Y_{\beta}<n$ for any $\beta \in \mathcal{R}$. We may assume that dimension of $X$ is at least three and thus by Grothendieck-Leftschetz theorem, we can identify $\operatorname{Pic}(X)$ and $\operatorname{Pic}(Y)$ by the restriction map. Let $H_{\beta}$ be the restriction of a divisor $\tilde{H}_{\beta}$ on $Y$. We claim that the divisor

$$
\tilde{H}:=\sum_{\beta \in \mathcal{R}} \tilde{H}_{\beta}
$$

is ample on $Y$. This is equivalent to $\tilde{H} \cdot \beta>0$ for all $\beta \in \mathcal{R}$. To see this, it suffices to prove the inequalities

$$
\left.\tilde{H}_{\beta}\right|_{\beta^{\prime}} \geq 0 \text { and }\left.\quad \tilde{H}_{\beta}\right|_{\beta}>0
$$

for any $\beta, \beta^{\prime} \in \mathcal{R}$. Since the fibers of $f_{\beta^{\prime}}: X \rightarrow Y_{\beta^{\prime}}$ have positive dimension, any curve $C$ contained in the fibers of $X \rightarrow Y_{\beta^{\prime}}$ has its class in $Y$ a non-zero multiple $N \beta^{\prime}$. The curve $C$ can be chosen to be movable in $X$ and thus we get

$$
N \tilde{H}_{\beta} \cdot \beta^{\prime}=H_{\beta} \cdot C \geq 0,
$$

because $H_{\beta}$ is effective in $X$. The second inequality follows from Lemma 3.5. Thus the claim is proved.

The divisor $H:=\left.\tilde{H}\right|_{X}$ is thus ample on $X$, hence it is connected and any point on divisors $H_{\beta}$ represents the same class $c_{X}=c_{H_{\beta}}$ in $\mathrm{CH}_{0}(X)$. 
Case 2: $\operatorname{dim} Y_{\beta}=n$ for some $\beta \in \mathcal{R}$. In this case, $\left.f_{\beta}\right|_{X}: X \rightarrow Y_{\beta}$ is a double cover (out of some codimension two subvariety, where $X$ contains a whole fiber of $f_{\beta}$ ). Due to Example 3.8, the divisor $H:=H_{\beta}$ is a pull-back of an ample divisor on $Y_{\beta}$, so $H$ meets $H_{\beta^{\prime}}$ for any $\beta^{\prime}$. It thus follows that $c_{H_{\beta^{\prime}}}=c_{H_{\beta}}$ for any $\beta^{\prime}$ and we can take $c_{X}$ as the class of a point on $H$.

Note that in a situation where $Y$ is not homogeneous, but still satisfies assumption $(\star)$ the following alternative lemma could be used.

Lemma 4.2. If there exist an ample divisor $\tilde{H}$ on $Y$ such that $\tilde{H} \cap X$ is a CCD on $X$ with associated class $c_{H}$, then for any curve $C$ on $Y,\left.C\right|_{X}$ is proportional to $c_{H}$ and, in particular, the intersection of any $n$ divisors is proportional to $c_{H}$.

Proof. We have the following commutative diagram:

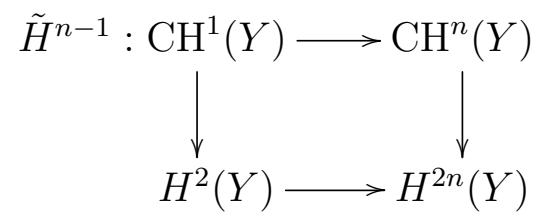

The vertical arrows are isomorphisms due to condition $(\star)$, the bottom arrow is also an isomorphism by the hard Lefschetz theorem, hence the top arrow is an isomorphism. Therefore the restriction $\left.C\right|_{X}$ is equal to $\left.\left(L \cdot \tilde{H}^{n-1}\right)\right|_{X}$ for some divisor $L$ on $Y$. But the last restriction can be represented by a zero-cycle with the support on $H$, hence is proportional to $c_{H}$.

Proof of Theorem 1.2. The divisor $H$ constructed in the proof of Lemma 4.1 does not intersect a constant cycle subvariety $Z$ only if $Z$ is a rational curve contracted by $f_{\beta}$ and $\operatorname{dim} Y_{\beta}=n$. But in this case the class of a point on $Z$ is proportional to $\left.\beta\right|_{X}$ and hence is proportional to $c_{X}$. Let $\tilde{D}_{i}$ be a divisor on $Y$ such that $\left.\tilde{D}_{i}\right|_{X}=D_{i}$ in $\operatorname{Pic}(X)$; we then have in $\mathrm{CH}_{0}(X)$

$$
D_{1} \cdot \ldots \cdot D_{n}=\left.\left(\tilde{D}_{1} \cdot \ldots \cdot \tilde{D}_{n}\right)\right|_{X} .
$$

The intersection in the right-hand side is a (reducible) curve in $Y$, we proved that the restriction of any curve in $Y$ to $X$ is proportional to $c_{X}$ in Proposition 4.1 .

The top Chern class of $X$ can be represented by a combination of Chern classes of $Y$ and Chern classes of line bundle $\mathcal{O}_{Y}(X)$. This is again a restriction of some (reducible) curve on $Y$. 
Remark 4.3. There is another strategy to prove Theorem $1.2 \mathrm{~b}$. If $\operatorname{dim} Y_{\beta}<n$ for some $\beta$ then

$$
D_{1} \cdot \ldots \cdot D_{n}=0
$$

for any divisors $D_{i}$ on $Y_{\beta}$. The same is true for divisors $\left.f_{\beta}^{*}\left(D_{i}\right)\right|_{X}$ on $X$. Since $H_{\beta}$ and $\left.f_{\beta}^{*}\left(\operatorname{Pic}\left(Y_{\beta}\right)\right)\right|_{X}$ generate $\operatorname{Pic}(X) \otimes \mathbb{Q}$, any intersection of $n$ divisors on $X$ is proportional to a zero-cycle with support on $H_{\beta}$ and hence is proportional to $c_{X}$.

Remark 4.4. The method could be presumably generalized to the case where $Y$ is a Fano variety satisfying assumption $(\star)$ using Lemma 4.2 .

\section{References}

[1] A. Beauville. On the splitting of the Bloch-Beilinson filtration. Algebraic cycles and motives (vol. 2), 344:38-53, 2007.

[2] A. Beauville and C. Voisin. On the Chow ring of a K3 surface. J. Alg. Geom, 13:417-426, 2004.

[3] M. Brion. Lectures on the geometry of flag varieties. 2004.

[4] M. Brion and S. Kumar. Frobenius Splitting Methods in Geometry and Representation Theory, volume 231 of Progress in Mathematics. Birkhäuser Basel, 2005.

[5] L. Fu. Beauville-Voisin conjecture for generalized Kummer varieties. International Mathematics Research Notices, 2014.

[6] D. Huybrechts. Curves and cycles on K3 surface. Algebraic Geometry, 1:69-106, 2014.

[7] H.-Y. Lin. On the chow group of zero-cycles of a generalized Kummer variety. arXiv:1507.05155v1, 2015.

[8] D. Mumford. Rational equivalence of zero-cycles in surfaces. $J$. Math. Kyoto Univ., 9:195-204, 1968.

[9] A. Roitman. Rational equivalence of zero-dimansional cycles. Mat. Sb. (N.S.), 89(131):569-585, 1972.

[10] A. Roitman. The torsion of the group of zero-cycles modulo rational equivalence. Ann. Math, 111:553-569, 1980. 
[11] C. Voisin. On the chow ring of certain algebraic hyper-Kähler manifolds. Pure Appl. Math. Q., 4(3):613-649, 2008.

[12] C. Voisin. Rational equivalence of zero-cycles on K3 surfaces and conjectures of Huybrechts and O'Grady. To appear in "Recent Advances in Algebraic Geometry, a conference in honor of Rob Lazarsfeld's 60th birthday", 2012.

[13] C. Voisin. Remarks and questions on coisotropic subvarieties and zero-cycles of hyper-Kähler varieties. arXiv:1501.02984, 2015 .

[14] G. Welters. Abel-Jacobi isogenies for certain types of Fano threefolds. Mathematical Centre Tracts, 141, 1981.

Institut de Mathématiques de Jussieu, 4 Place Jussieu, 75005 France, ibazhov@gmail.com 